\title{
atonal- and achaete-scute-related genes in the annelid Platynereis dumerilii: insights into the evolution of neural basic-Helix-Loop-Helix genes
}

\author{
Elena Simionato ${ }^{1}$, Pierre Kerner ${ }^{1}$, Nicolas Dray ${ }^{1}$, Martine Le Gouar ${ }^{1}$, \\ Valérie Ledent ${ }^{2}$, Detlev Arendt ${ }^{3}$ and Michel Vervoort*1,4
}

Address: ${ }^{1}$ Evolution et Développement des métazoaires, Centre de Génétique Moléculaire-UPR 2167 CNRS, 1, av. de la terrasse, 91198 Gif-surYvette, France, ${ }^{2}$ Belgian EMBnet Node - Laboratoire de Bioinformatique, Université Libre de Bruxelles, Institut de Biologie et de Médecine Moléculaires, Rue des Professeurs Jeener et Brachet 12, B-6041 Gosselies, Belgium, ${ }^{3}$ Developmental Biology Unit, European Molecular Biology Laboratory, 69117 Heidelberg, Germany and ${ }^{4}$ UFR Sciences du Vivant, Université Paris Diderot - Paris 7, 5, rue Marie-Andrée Lagroua Weill-Hallé, 75205 Paris Cedex 13, France

Email: Elena Simionato - simionato@cgm.cnrs-gif.fr; Pierre Kerner - kerner@cgm.cnrs-gif.fr; Nicolas Dray - ndray@cgm.cnrs-gif.fr; Martine Le Gouar - legouar@cgm.cnrs-gif.fr; Valérie Ledent - valerie.ledent@ulb.ac.be; Detlev Arendt - arendt@embl.de;

Michel Vervoort* - vervoort@cgm.cnrs-gif.fr

* Corresponding author

Published: 9 June 2008

BMC Evolutionary Biology 2008, 8: 170 doi:10.1/86//47|-2|48-8-170
Received: I April 2008

Accepted: 9 June 2008

This article is available from: http://www.biomedcentral.com/I47I-2/48/8/I70

(C) 2008 Simionato et al; licensee BioMed Central Ltd.

This is an Open Access article distributed under the terms of the Creative Commons Attribution License (http://creativecommons.org/licenses/by/2.0), which permits unrestricted use, distribution, and reproduction in any medium, provided the original work is properly cited.

\begin{abstract}
Background: Functional studies in model organisms, such as vertebrates and Drosophila, have shown that basic Helix-loop-Helix (bHLH) proteins have important roles in different steps of neurogenesis, from the acquisition of neural fate to the differentiation into specific neural cell types. However, these studies highlighted many differences in the expression and function of orthologous bHLH proteins during neural development between vertebrates and Drosophila. To understand how the functions of neural bHLH genes have evolved among bilaterians, we have performed a detailed study of bHLH genes during nervous system development in the polychaete annelid, Platynereis dumerilii, an organism which is evolutionary distant from both Drosophila and vertebrates.

Results: We have studied Platynereis orthologs of the most important vertebrate neural bHLH genes, i.e. achaete-scute, neurogenin, atonal, olig, and NeuroD genes, the latter two being genes absent of the Drosophila genome. We observed that all these genes have specific expression patterns during nervous system formation in Platynereis. Our data suggest that in Platynereis, like in vertebrates but unlike Drosophila, (i) neurogenin is the main proneural gene for the formation of the trunk central nervous system, (ii) achaetescute and olig genes are involved in neural subtype specification in the central nervous system, in particular in the specification of the serotonergic phenotype. In addition, we found that the Platynereis NeuroD gene has a broad and early neuroectodermal expression, which is completely different from the neuronal expression of vertebrate NeuroD genes.

Conclusion: Our analysis suggests that the Platynereis bHLH genes have both proneural and neuronal specification functions, in a way more akin to the vertebrate situation than to that of Drosophila. We conclude that these features are ancestral to bilaterians and have been conserved in the vertebrates and annelids lineages, but have diverged in the evolutionary lineage leading to Drosophila.
\end{abstract}




\section{Background}

Neurogenesis is a complex process that involves the formation of a vast array of neuronal and glial cell types that must be produced in the correct numbers and at appropriate positions. Genetic and molecular studies mainly conducted in Drosophila and vertebrates have shown that genes encoding transcription factors of the basic HelixLoop-Helix (bHLH) class play pivotal roles in various steps of neurogenesis, including commitment of neural precursors (proneural function), specification of particular neuronal identities, and neuronal differentiation [1-5]. Most of the genes encoding bHLH transcription factors and which are involved in neural development (hereafter named neural bHLH genes), belong to five of the numerous phylogenetically-defined bHLH families, achaete-scute and four families of atonal-related genes, neurogenin, atonal, olig (oligo), and NeuroD [2,6]. While some of the neural bHLH genes show strikingly similar functions in Drosophila and vertebrates $[2,3,7]$, there are also profound differences between them $[1,4]$.

First, in vertebrates, genes of the neurogenin family (ngn1, $n g n 2$, and $n g n 3$ ) are required for the formation of the precursors of many neural cells of both the Peripheral and Central Nervous Systems (PNS and CNS) [8-10] while their single Drosophila ortholog, tap/biparous, has no proneural role and is expressed in a few differentiating neural cells $[11,12]$. In Drosophila, the main proneural bHLH genes for the CNS belong to the achaete-scute family and are also involved, together with atonal family genes, in the formation of the sensory organs $[2,3,13,14]$. Vertebrate achaete-scute and atonal genes probably also have proneural functions but in a much more limited set of cells, in particular in the CNS $[1,2,4]$.

Second, vertebrate proneural genes contribute to the specification of progenitor-cell identity $[2,4,5]$. A clear example of such a function is provided by the dorsal embryonic spinal cord, in which Math1 (atonal family), ngn1, and Mash1 (ascl1; achaete-scute family) are required for the correct specification of discrete dorsoventral progenitor domains that produce distinct types of interneurons [1518]. Mash1 has also been shown to have instructive roles in the specification of noradrenergic, GABAergic, and serotonergic neurons in various positions in the brain and the spinal cord [19-22]. Finally, ngn2 has a key role for motor neurons formation in the ventral spinal cord $[23,24]$. Such important roles in neuronal specification for proneural bHLH genes in the CNS are not found in Drosophila $[1,4]$.

Third, bHLH genes that have important functions during vertebrate neurogenesis do not have orthologs in Drosophila. Many vertebrate neurons require the function of genes, which belong to the NeuroD family, for their proper differentiation and survival [25-27]. Genes of the olig family (olig1, olig2, and olig3) have key roles in the specification of motor neurons, dorsal interneurons, and oligodendrocytes in the vertebrate CNS [18,23,24,28-30]. olig and NeuroD genes do not exist in Drosophila (the gene known in Drosophila as Dm-oli is in fact an ortholog of the vertebrate Beta3 genes) [6,31].

Given these differences and in order to decipher which aspects of the functions of neural bHLH genes are ancestral to bilaterians and which are derived characters specific to some bilaterian lineages, we have isolated and studied these genes in the polychaete annelid, Platynereis dumerilii, which belongs to a different branch (Trochozoa) of the bilaterians tree than Drosophila (Ecdysozoa) and vertebrates (Deuterostoma) and is therefore evolutionary distant to both these organisms [32]. In addition, Platynereis is considered to have retained some bilaterian ancestral features, making it a useful model for comparative developmental biology [e.g. [33-35]]. Here, we report the expression patterns of Platynereis orthologs of the most important neural bHLH genes, including the olig and NeuroD genes not found in Drosophila. Our data suggest that Platynereis bHLH genes have both proneural and neuronal specification functions, in a way more akin to the vertebrate situation than to that of Drosophila. These data indicate that these functions were already established in Urbilateria, the last common ancestor of all bilaterians.

\section{Results \\ Brief overview of the formation of the Platynereis larval nervous system}

Platynereis displays an indirect development which gives rise to a ciliated trochophore larva that subsequently metamorphoses into a juvenile worm [36]. The formation of the Platynereis larval trunk nervous system has been thoroughly described, up to the late trochophore stage (metatrochophore; 48 to 55 hours post fertilization, hpf), using whole-mount in situ hybridization (WMISH) with RNA antisense probes corresponding to Platynereis neuronal differentiation genes, such as elav (Pdu-elav), synaptotagmin (Pdu-syt), Tryptophane Hydroxylase (Pdu-TrpH), and Vesicular Acetylcholine Transporter (Pdu-VAchT) [35]. In order to give an overview of larval neurogenesis, which is important to understand the next parts of this article, we show here some WMISH for these previously characterized genes in $24 \mathrm{hpf}$ to $55 \mathrm{hpf}$ larvae and extend the published description by looking at juvenile worms (72 hpf).

A simple larval nervous system first differentiates during the early ( $24 \mathrm{hpf}$ ) to late trochophore ( $48 \mathrm{hpf}$ ) stages: a few cells expressing Pdu-elav are observed on the ventral side of the $24 \mathrm{hpf}$ and $34 \mathrm{hpf}$ larvae (Figure 1A,B) and these cells give rise to two bilateral anterior groups and one posterior group of $P d u$-syt-expressing neurons (Figure 

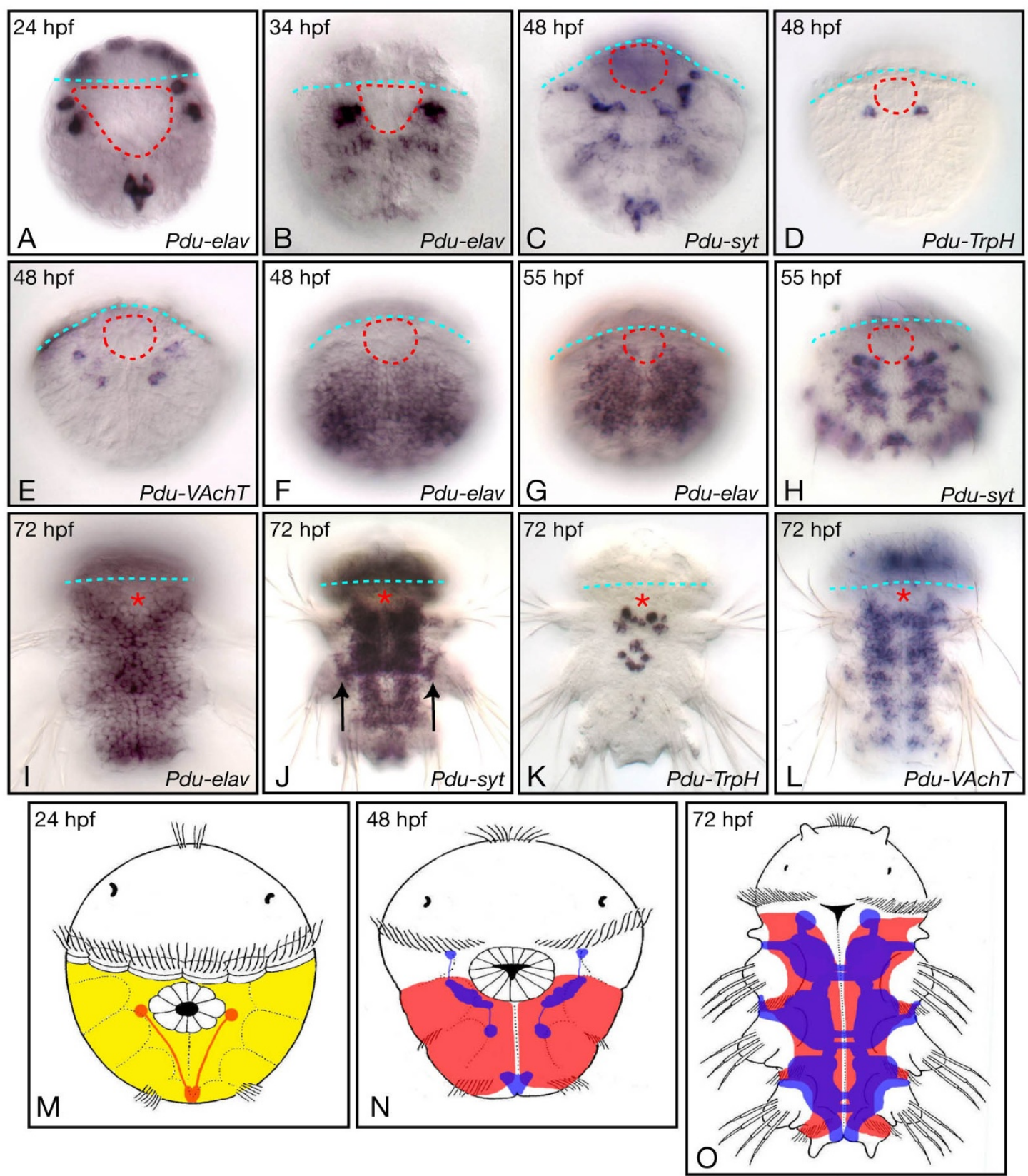

\section{Figure I}

Overview of formation the trunk nervous system in Platynereis. Expression of Pdu-elav, Pdu-syt, Pdu-TrpH, and Pdu$V A c h T$, as determined by WMISH, on a selection of larval stages is shown. All pictures are ImageJ projections and are ventral views (anterior up). The blue dotted lines indicate the position of the prototroch (a ring of ciliated cells involved in the locomotion of the larva) and therefore the separation between the prospective head (up) and trunk (down) regions. The position of the mouth region is indicated either by dotted red lines or a red asterisk, depending on the stage. The formation of the larval neurons is shown in A to E. A few neurons with stereotyped positions differentiate between $24 \mathrm{~h}$ to $48 \mathrm{~h}$ as seen by the expression of Pdu-elav and Pdu-syt (A-C). A single pair of neurons, located close to the mouth region, is serotonergic as seen by the expression of Pdu-TrpH (D), a few other neurons are cholinergic (as seen by the expression of Pdu-VAchT; E), and the other ones are of unknown identity. $F$ to $L$ depict the formation of the juvenile worm nervous system. See the main text for details. Arrows in J point to peripheral neurons associated with the parapodes. $M$ to $O$ are schematic drawings of the organization of the nervous system of $24 \mathrm{hpf}, 48 \mathrm{hpf}$, and $72 \mathrm{hpf}$ larvae. The neuroectodermal cells are indicated in yellow, the cells expressing Pdu-elav in red, and the Pdu-syt-expressing neurons in blue. 
1C). Different neuronal subtypes can be identified, such as serotonergic (identified as cells expressing $P d u-T r p H$ ) and cholinergic (identified as cells expressing Pdu-VAchT; Figure 1D,E). From 48 hpf, a large number of cells enter neural differentiation and express first Pdu-elav (Figure $1 \mathrm{~F}, \mathrm{G}$ ) and then $P d u$-syt (Figure $1 \mathrm{H}$ ). These neurons form the ventral nerve cord (VNC) of the $72 \mathrm{hpf}$ juvenile worm (Figure 1I,J). Many of the formed neurons are cholinergic neurons as seen by the massive expression of Pdu-VAchT (Figure 1L) in the VNC, except in its medialmost part where several serotonergic neurons differentiate (expression of $P d u$-TrpH; Figure $1 \mathrm{~K}$ ). Outside the VNC, peripheral neurons are also found mainly associated with the appendages (the parapodes; arrows in Figure 1J).

\section{Identification of Platynereis atonal- and achaete-scute- related $b H L H$ genes}

By sequence similarity searches on an expressed sequence tag (EST) collection (40,000 ESTs from normalized cDNA libraries of mixed larval stages, corresponding to more than 10,000 unigene clusters) [34], we identified several Platynereis dumerilii bHLH genes among which some show strong sequence similarity to either achaete-scute- or atonal-related genes. The predicted amino acid sequence of the bHLH domains were aligned with those of a sample of metazoan bHLH genes identified in a previous study [31]. Multiple phylogenetic reconstructions show that we identified orthologs of the neurogenin/biparous, achaetescute/ASCL/ASH, olig, and NeuroD genes (Figures 2 and 3). We named these genes Pdu-Ngn, Pdu-ASH, Pdu-Olig, and $P d u-N e u r o D$, respectively. For each family, we found a single Platynereis member but we cannot exclude that additional members do exist, although duplicated evolutionary-conserved genes are rare in Platynereis [34]. We also included in our analysis the previously identified atonal ortholog ( $P d u-A T H$; not found in the EST collection) $[35,37]$ in order to study Platynereis representatives for all the main families of bHLH genes involved in neural determination and specification in vertebrates and Drosophila $[2,4]$. We also identified Beta3 and mist genes in Platynereis (Figure 2), but these genes were not further characterized as their Drosophila and vertebrate orthologs do not have well defined functions in neurogenesis. We used WMISH to monitor the expression of Pdu-Ngn, PduASH, Pdu-Olig, Pdu-ATH, and Pdu-NeuroD during Platynereis development and focused on possible expressions during trunk nervous system formation for which a good characterization exists [35]. All the genes are also expressed in the head, probably in the brain and/or sensory organs, but these expressions were not further characterized.

\section{Platynereis atonal- and achaete-scute-related bHLH genes are expressed during trunk nervous system formation}

The five bHLH genes are already expressed in 24 hpf larvae. $P d u-N e u r o D$ is expressed in a broad ventral ectodermal domain (Figure 4A) which corresponds to the larval neuroectoderm. This expression domain is similar to that of Platynereis orthologs of bilaterian conserved neuroectodermal genes, such as SoxB (Figure 5A-C and P.K. et al., unpublished). Pdu-Ngn and Pdu-ASH are expressed in a few cells located on the ventral side of the larvae, in particular around the stomodaeal area (Figure 4B,C). This expression nicely prefigures the distribution of differentiating neurons that is observed in slightly later stages (Figure $1 \mathrm{~B}, \mathrm{C})$, suggesting that the two genes are expressed in precursors of the larval central nervous system. Pdu-ATH and $\mathrm{Pdu}$-Olig are expressed in a few lateral cells we were unable to identify (Figure 4D,E).

In 34 hpf larvae, Pdu-NeuroD is still expressed in a broad ventral ectodermal domain (Figure 4F) which includes the prospective VNC region in which $P d u-N g n$ and $P d u-A S H$ become widely expressed (Figure $4 \mathrm{G}, \mathrm{H}$ ). At this stage, the expression of $P d u$-SoxB strongly decreases in the same region (Figure 5D). In vertebrates, the transition from neuroectodermal cells to committed progenitors is linked to the activation of the expression of bHLH proneural genes and a concomitant repression of the expression of SoxB genes [e.g. [2,38]]. Our data suggest that a similar transition occurs around $34 \mathrm{hpf}$ in the Platynereis larvae. However, at this stage, only very few cells express Pdu-elav (Figure 1B), indicating that the Pdu-Ngn and $P d u-A S H$ expressing precursors are still not engaged towards differentiation. Both Pdu-Ngn and Pdu-ASH are also expressed in more lateral cells that may correspond to peripheral nervous system precursors (Figure 4G,H). Pdu-ATH and $\mathrm{Pdu}$-Olig continue to be expressed in lateral cells, with $P d u$-Olig expressed in three bilateral small stripes (Figure $4 \mathrm{I}, \mathrm{J})$.

In $40 \mathrm{hpf}$ to $55 \mathrm{hpf}$ larvae, Pdu-NeuroD continues to be broadly expressed in the ventral ectoderm (Figure $4 \mathrm{~K}, \mathrm{P}$ ). Expression of Pdu-NeuroD is restricted to the superficial layer of the ectoderm (not shown). From $40 \mathrm{hpf}$ to $55 \mathrm{hpf}$ larvae, $P d u-N g n$ is largely expressed in the whole prospective $\mathrm{VNC}$ region as well as in some more lateral cells (Figure $4 \mathrm{~L}, \mathrm{Q})$. Pdu-Ngn is expressed in a salt and pepper manner, with highly-expressing cells interspersed with weakly-expressing ones. In $40 \mathrm{hpf}$ and $48 \mathrm{hpf}$ larvae, Denes et al. [35] showed that the prospective Platynereis $\mathrm{VNC}$ region is multilayered with, from superficial (apical) to more internal (basal), a proliferating progenitor zone (a single layer of BrdU incorporating cells), a post-mitotic progenitor zone (Pdu-elav positive, Pdu-syt negative cells), and a differentiation zone (Pdu-elav positive, Pdu-syt positive cells). To define in which zone(s) of the prospective 




Figure 2

Phylogenetic analysis of the Platynereis atonal-related bHLH genes. The phylogenetic tree has been constructed by Maximum Likelihood (ML) as described in the Methods section. Similar tree topologies were obtained using other phylogenetic reconstruction methods (not shown). The different groups of orthology are indicated (for more details, see [3I]). Statistical supports for the internal branches that define these groups are indicated (first number: bootstrap support in Neighbour-joining (NJ) analysis; second number: bootstrap support in ML analysis; third number: posterior probability in Bayesian inference analysis). The tree has been rooted using a non-atonal-related (twist) bHLH gene as outgroup. Platynereis genes are indicated in bold red. Species abbreviations: Cc: Capitella spl (annelid); Dm: Drosophila melanogaster (insect); Dp: Daphnia pulex (crustacean); Lg: Lottia gigantea (mollusk); Mm: Mus musculus (vertebrate); Pdu: Platynereis dumerilii (annelid); Sp: Strongylocentrotus purpuratus (echinoderm); Tc: Tribolium castaneum (insect). 


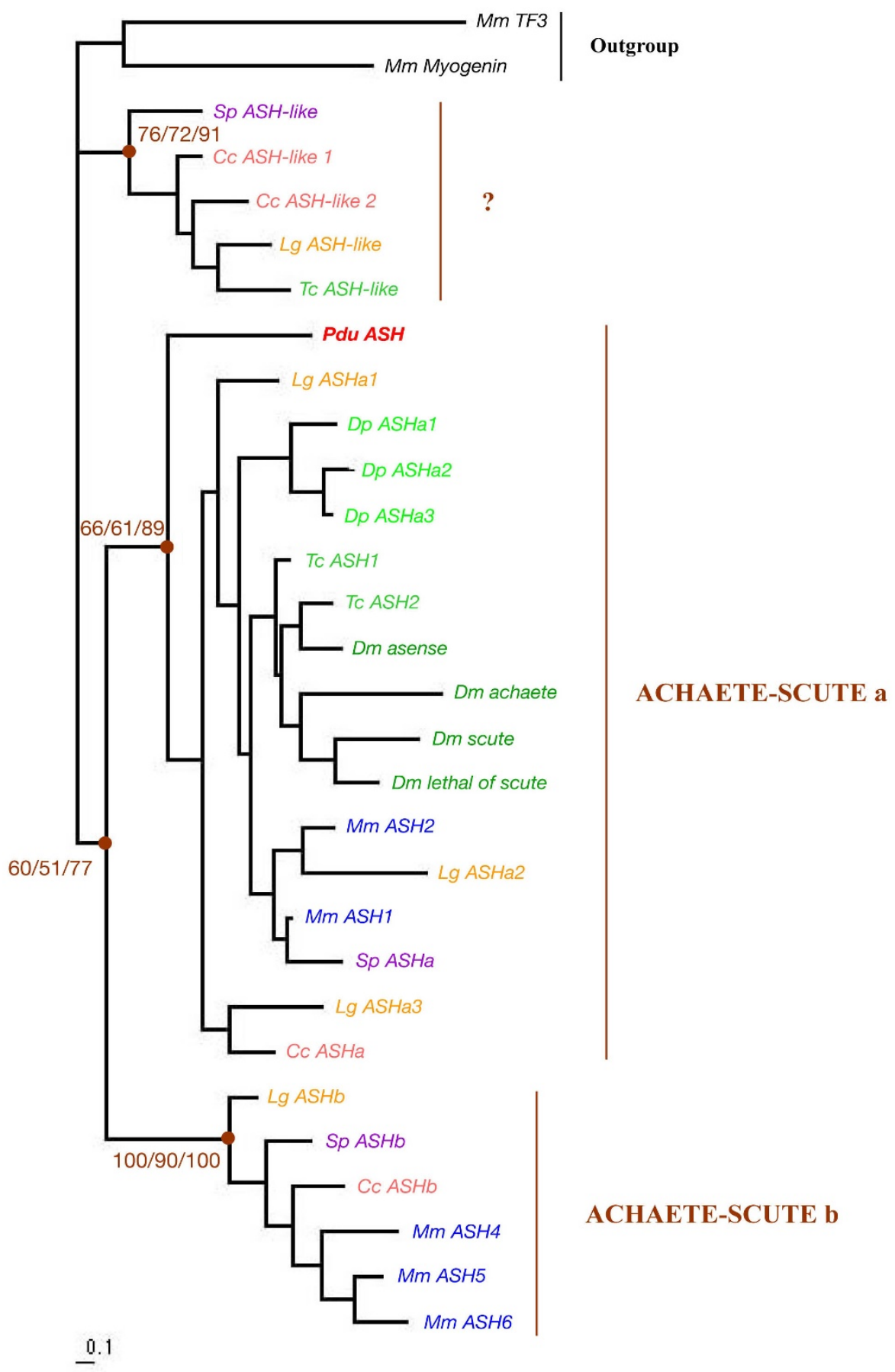

Figure 3

Phylogenetic analysis of the Platynereis achaete-scute-related bHLH gene. The phylogenetic tree has been constructed by Maximum Likelihood (ML) as described in the Methods section. Similar tree topologies are obtained using other phylogenetic reconstruction methods (not shown). Statistical supports for the internal branches that define these groups are indicated (first number: bootstrap support in Neighbour-joining (NJ) analysis; second number: bootstrap support in ML analysis; third number: posterior probability in Bayesian inference analysis). The tree has been rooted using non-achaete-scute bHLH genes as outgroup. The Platynereis gene is indicated in bold red. The "?" indicates a group of divergent achaete-scute-like genes found in some species and that cannot be related to either of the two bilaterian achaete-scute families (see [3I] for more details). Species abbreviations are as in Figure 2. 


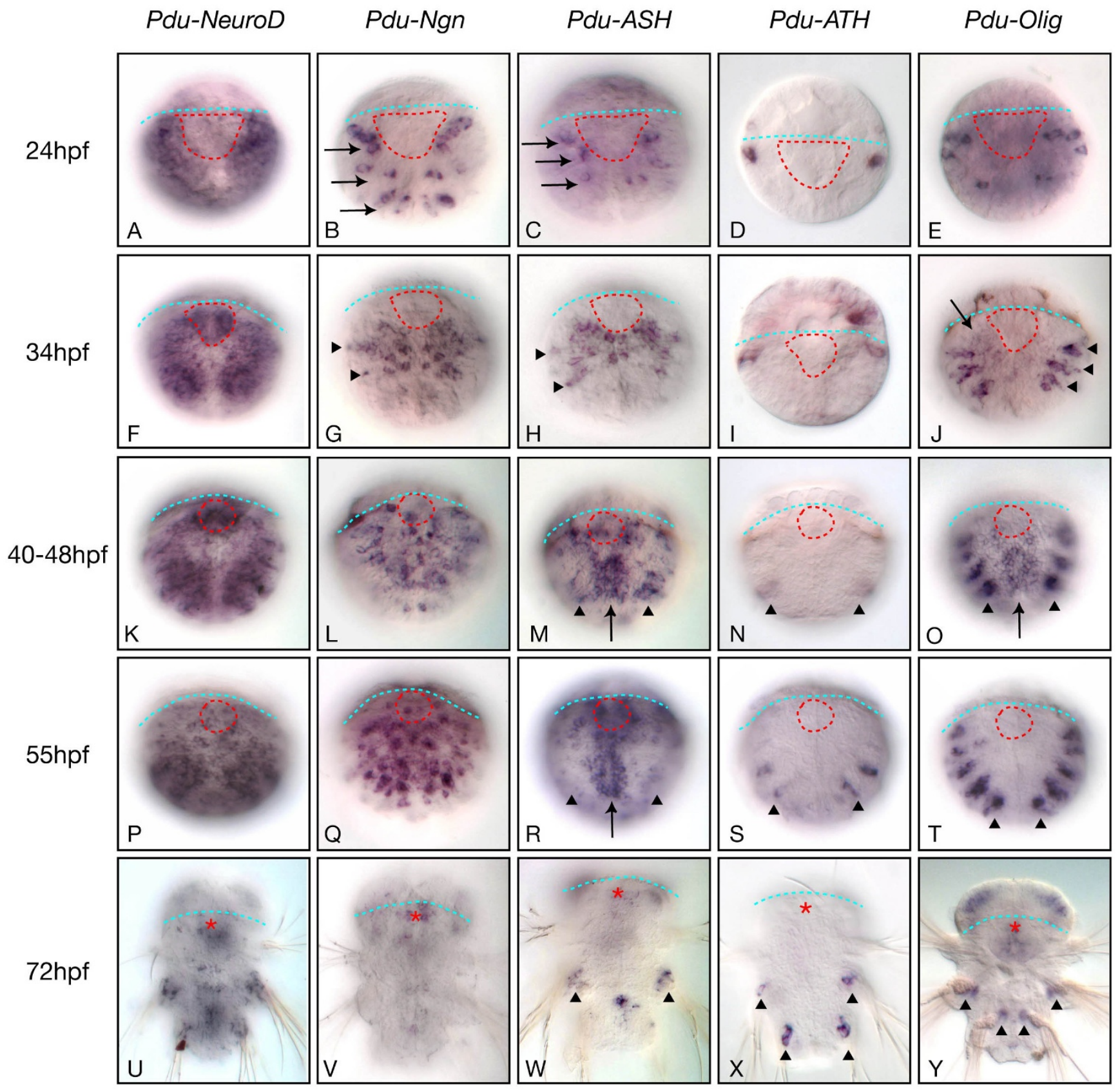

Figure 4

Expression of Platynereis neural bHLH genes during trunk neurogenesis. Expression of Pdu-NeuroD, Pdu-neurogenin (Pdu-Ngn), Pdu-achete-scute (Pdu-ASH), Pdu-Olig, and Pdu-atonal (Pdu-ATH), as determined by WMISH, on a selection of larval stages is shown. Most of the images are ImageJ projections and all images are ventral views (anterior up). Labels are as in Figure I. See the main text for the detailed descriptions of the expression patterns. In (B) and (C), arrows point to the groups of cells expressing Pdu-Ngn or Pdu-ASH and whose positions correspond to the neurons that will latter differentiate (compare with Figure $I B, C)$. In $(G)$ and $(H)$, the arrowheads point to putative PNS precursor cells. In $(J)$, the arrow points to a weak medial expression of Pdu-Olig and arrowheads to its more lateral expression domains that form three bilateral small stripes. In (M, $N$, $\mathrm{O}, \mathrm{R}, \mathrm{S}, \mathrm{T})$, the arrow points to the medial expression and the arrowheads to the more lateral expression domains that lie outside the VNC. In (W-Y), arrowheads point to expressions in cells associated with the parapodes. Labelling associated with the parapodes in $(U)$ is probably background (as seen at higher magnification). 


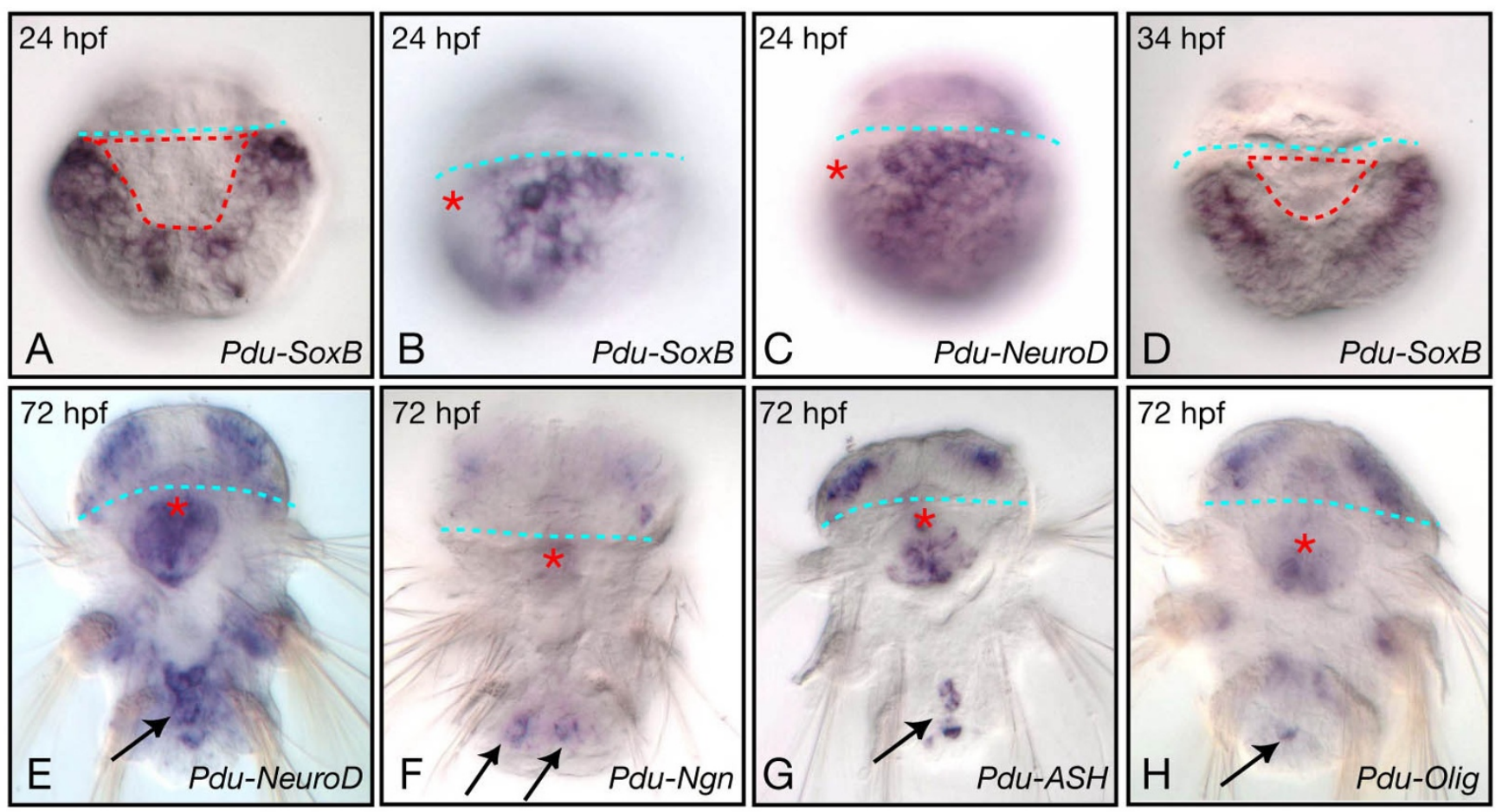

Figure 5

Additional expressions of Platynereis neural genes during trunk neurogenesis. $A, D, E-H$ are ventral views; $B, C$ are lateral views (ventral on the left). Most of the images are ImageJ projections. Labels are as in Figure I. E-H correspond to more internal views than those of Figure $4 \mathrm{U}-\mathrm{Y}$ which are superficial views. See the main text for details. Arrows in E-H point to internal cells expressing neural bHLH genes and that may belong to the posterior growth zone [4I,42].

VNC Pdu-Ngn is expressed, we performed double WMISH [39] for Pdu-Ngn, on one hand, and Pdu-elav or Pdu-syt, on the other hand, and visualized the labellings with confocal microscopy (Figure 6). We found that Pdu-Ngn is mainly expressed in superficial cells in contrast to Pdu-elav and Pdu-syt (Figure 6A,B). We used 3D reconstructions of confocal stacks to perform virtual cross-sections of the VNC region (Figure $6 \mathrm{~F}, \mathrm{H}$ ): we found that $\mathrm{Pdu}-\mathrm{Ngn}$ is expressed in the apicalmost cells of the prospective VNC with very little overlap with Pdu-elav and no overlap with $P d u$-syt. We therefore conclude that Pdu-Ngn is expressed in undifferentiated neural precursors and mainly in the proliferating ones.

Pdu-ASH and Pdu-Olig, at 40 and $48 \mathrm{hpf}$, are expressed in the medialmost part of the prospective $\mathrm{VNC}$ region as well as in more lateral cells (Figure $4 \mathrm{M}, \mathrm{O}$ ). As for $\mathrm{Pdu}-\mathrm{Ngn}$, $\mathrm{Pdu}-\mathrm{ASH}$ and $\mathrm{Pdu}$-Olig are expressed in proliferating progenitors, as determined by double WMISH (Figure 6C,D,G,I and not shown). The expression of Pdu-ASH and $\mathrm{Pdu}$-Olig in the medialmost part of the prospective VNC region is similar to that of the Platynereis NK2.2 gene and corresponds to the region from which serotonergic neurons will emerge (Figure 1K) [35]. We confirmed by dou- ble WMISH that the serotonergic neurons $(\mathrm{Pdu}-\mathrm{Tr} p H-$ expressing cells) are located below the Pdu-ASH-expressing precursors (Figure 6J). At $55 \mathrm{hpf}, \mathrm{Pdu}$-ASH shows the same expression pattern than in the previous stages, but $\mathrm{Pdu}$-Olig is no more expressed in the prospective VNC region (Figure 4R,T). In $40 \mathrm{hpf}$ to $55 \mathrm{hpf}$ larvae, $P d u$-ATH is only expressed in a few cells located on both sides of the prospective VNC region (Figure $4 \mathrm{~N}, \mathrm{~S}$ ).

At $72 \mathrm{hpf}$, the five genes are expressed in more limited sets of cells in the VNC region and in cells associated with the parapodes (Figure $4 \mathrm{U}-\mathrm{Y}$ ). In addition, $\mathrm{Pdu}-\mathrm{Ngn}, \mathrm{Pdu}-\mathrm{ASH}$, $P d u$-Olig, and Pdu-NeuroD are expressed in posteriorlylocated internal cells (Figure 5E-H) whose position roughly corresponds to that of cells expressing Platynereis hunchback [40] and stem-cells markers, such as Platynereis piwi and vasa [41]. The cells expressing Pdu-Ngn, Pdu-ASH, Pdu-Olig, and Pdu-NeuroD may therefore belong to the posterior subterminal growth zone that will allow the posterior addition of new segments to the existing ones (and the corresponding elongation of the VNC) in a sequential manner throughout most of the life of the animal $[41,42]$. 

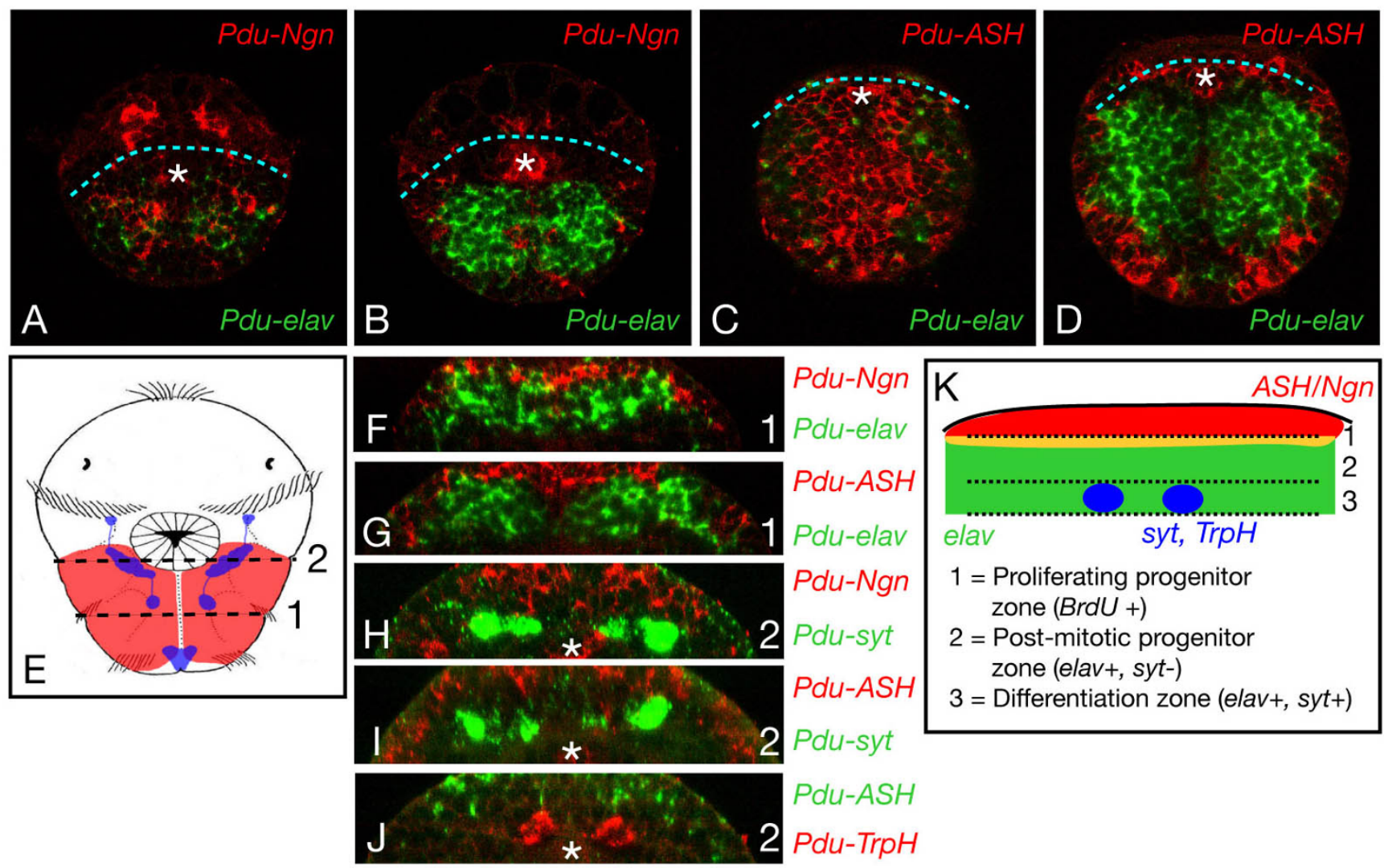

Figure 6

Position of the Pdu-Ngn- and Pdu-ASH-expressing cells with respect to the apicobasal organization of the prospective ventral nerve cord region. Confocal picures of double WMISH are shown. The probes which have been used are indicated on the pictures. A and $C$ are superficial plans, B and D more internal ones. Pdu-Ngn and Pdu-ASH are mainly expressed in superficial cells while Pdu-elav is mainly expressed in more profound cells. $F$ to $\mathrm{J}$ are virtual cross-sections, sections have been made at two anteroposterior levels ( $I$ and 2) as indicated on a schematic drawing (E). Apical is up, basal is down. Pdu-Ngn and Pdu-ASH are mainly expressed in the apicalmost layer of cells of the prospective VNC while Pdu-elav, Pdu-syt, and Pdu-TrpH are expressed in more basal cells. Some internal cells also express Pdu-Ngn $(H)$ and Pdu-ASH (not shown) and on some confocal sections we can see rare cells co-expressing Pdu-Ngn or Pdu-ASH and Pdu-elav. Such co-expressions are never observed with Pdu-syt or Pdu-TrpH. The white asterisk indicates an expression in the stomodeum. $\mathrm{K}$ summarizes the apicobasal layering of the Platynereis prospective VNC based on the data published by Denes et al. [35] and includes the expression of Pdu-Ngn and PduASH defined in this study. Apical is up, basal is down. Pdu-elav expression domain is in green, Pdu-ASH and Pdu-Ngn expression domain is in red, and the yellow region indicates the zone of overlap of these expression domains.

\section{Discussion}

Olig and NeuroD genes belong to the ancestral bilaterian toolkit of neural developmental genes

In this article, we report, for the first time, the characterization of several atonal- and achaete-scute-related bHLH genes from a lophotrochozoan species, the annelid Platynereis dumerilii. Our phylogenetic analyses demonstrate that we have identified neurogenin, achaete-scute, olig, and NeuroD orthologs in addition to a previously characterized atonal gene (Figures 2 and 3). neurogenin, achaetescute, and atonal genes have been found in many diverse species, including Drosophila melanogaster, Caenorhabditis elegans, and several vertebrates, and shown to be involved in neural development in all these species [e.g. $[2,7,31]]$. We found a similar situation in Platynereis as the three genes display specific expressions during neurogenesis (see below for further discussion), confirming their evolutionary-wide implication in neurogenesis in bilaterian animals.

NeuroD genes have been shown to be important neuronal differentiation genes in vertebrates (see introduction). A putative NeuroD gene (named cnd-1 but whose orthology relationship with the vertebrate NeuroD genes is only poorly supported; [43]) has been described in the nematode Caenorhabditis elegans and is involved in several steps 
of the formation of the motor neurons [44]. NeuroD genes cannot be found in the genomes of Drosophila melanogaster and of the urochordate Ciona intestinalis $[6,45]$. Our phylogenomic analysis (Figure 2) indicates that NeuroD genes are in fact widely found in bilaterians and that their absence in Drosophila melanogaster and Ciona intestinalis results from a rather specific loss in these species. We found the Platynereis NeuroD gene to have a broad and early neuroectodermal expression, which is completely different from the neuronal expression of vertebrate $\mathrm{Neu}$ roD genes and the neural subtype-specific expression of Caenorhabditis cnd-1. It is therefore difficult to infer from these data any putative ancestral expression or function of NeuroD. More precise inference will await additional data from other species.

Olig genes were thought to be vertebrate-specific genes as they are not found in the Drosophila melanogaster, Caenorhabditis elegans, and Ciona intestinalis genomes $[6,45]$. In fact, olig genes are found in several non-vertebrate species (Figure 2). However, we cannot find such genes in any of the sequenced arthropod and nematode genomes [[31], E.S. and M.V., unpublished observations], suggesting that olig genes have been lost quite early during the evolution of these phyla. We found the Platynereis olig gene to be specifically expressed during nervous system formation and that its expression shows similarities to that of vertebrate olig genes (see below). We therefore conclude that olig, together with neurogenin, achaete-scute, atonal, and NeuroD genes belong to the ancestral bilaterian toolkit of neural developmental genes.

\section{Vertebrate-like expression of Platynereis neurogenin suggests a major proneural role and provides insights into the evolution of the proneural function in bilaterians}

One striking difference between vertebrate and Drosophila neurogenesis is the differential use of neurogenin genes in these species. Indeed, neurogenin genes are the key proneural genes in the vertebrate CNS while it is not the case for their Drosophila counterpart (see introduction). In Drosophila, and probably more generally in arthropods, the proneural function in the CNS is mainly performed by the achaete-scute genes (see introduction and [46]). These genes have a much more limited proneural functions in vertebrates (see introduction). Given these differences, it is challenging to infer what was the ancestral situation, i.e. what were the main proneural genes acting in Urbilateria, the last common ancestor of all bilaterians. Our data on Platynereis help to answer this question.

We found that Platynereis neurogenin (Pdu-Ngn) has an expression suggestive of a wide proneural function in the developing trunk CNS which is similar to that of the vertebrate neurogenin genes. Indeed, Pdu-Ngn expression arises at early stages of neural development, overlapping that of the neuroectodermal marker SoxB, and preceding the expression of the differentiation marker elav. The expression domain of $\mathrm{Pdu}-\mathrm{Ngn}$ is large and covers the whole prospective CNS region (ventral part of the trunk ectoderm) as well as more lateral regions which probably correspond to the PNS. Pdu-Ngn is expressed in a salt and pepper manner which is often found for proneural genes as a consequence of lateral inhibition processes (reviewed in $[1,2])$. Finally, Pdu-Ngn is expressed in the apicalmost part of the forming CNS, which has been shown to include proliferative neuroectodermal cells and neural precursors [35]. $\mathrm{Pdu}-\mathrm{Ngn}$ is therefore expressed at the right time, the right place, and the right manner to be the major proneural gene for the formation of the Platynereis trunk nervous system, like its orthologs in vertebrates. This similarity between vertebrates (deuterostomes) and an annelid (a protostome) suggests that the broad proneural function of neurogenin genes is ancestral to bilaterians and has been lost in the evolutionary lineage leading to present-day insects (or even arthropods). This does not necessarily mean that proneural function of achaete-scute genes is a derived character: indeed, such function is found in vertebrates and in arthropods, and the expression of $P d u-A S H$ is not incompatible with this function, as it is expressed in early stages of VNC formation (although only in a subset of the Pdu-Ngn-expressing cells - an expression that is, to our opinion, more consistent with a role in neural specification). It is therefore conceivable that both neurogenin and achaete-scute were acting as proneural genes in Urbilateria, the former being the predominant one.

\section{Platynereis bHLH genes may have neural specification functions in the CNS - evolution of neural specification in bilaterians}

Another striking difference between vertebrates and Drosophila is the differential use of bHLH genes in neural subtype specification in the CNS. In vertebrate, neural bHLH genes such as olig and achaete-scute genes are important to specify many types of neural cells which are produced in the CNS, while it is not the case in the Drosophila CNS (although a single study has suggested that achaete-scute genes may be involved in the specification of a limited set of cells in the CNS [47]; but see [1]). This raises the possibility that the functions of bHLH genes in neural subtype specification may largely represent vertebrate innovations. Alternatively, they could be bilaterian ancestral functions that have been lost in the evolutionary lineage leading to Drosophila. We found that two Platynereis bHLH genes, $\mathrm{Pdu}$-ASH and $\mathrm{Pdu}$-Olig, are expressed in a way suggestive of an involvement in neural specification in the CNS. Both genes are expressed in the medialmost part of the CNS, they are first co-expressed and in a second time, only $P d u-A S H$ continues to be expressed. Interestingly, these expression profiles coincide with those of verte- 
brates olig and achaete-scute genes which are also expressed in medial parts of the neural tube (reviewed in [5]). Importantly, this similarity is probably meaningful as the overall organization of the Platynereis CNS bears striking resemblances with that of vertebrates, as seen by the conserved expression domains of many neural patterning and differentiation genes between Platynereis and vertebrates [35]. The cells expressing Pdu-ASH and Pdu-Olig correspond to a domain of the VNC characterized by the coexpression of Platynereis NK2.2 and NK6 genes [35]. In vertebrates, such as mammals, Ascl1/Mash1 is also expressed in a NK2.2/NK6 positive medial domain (p3 domain in the spinal cord and its topological equivalent in the hindbrain, PMNv) and controls the formation of serotonergic neurons from this domain in the hindbrain $[21,22]$. Strikingly, serotonergic neurons in Platynereis also emerge from a medial domain of the CNS, corresponding to cells expressing NK2.2 and achaete-scute. We therefore suggest that $P d u-A S H$ is involved in the specification of serotonergic neurons in Platynereis and that this may represent an ancestral function of achaete-scute genes in bilaterians.

In vertebrates, such as mammals, olig genes have important roles in neural specification. olig2 gene is expressed in the so-called pMN domain of the spinal cord, located slightly more laterally than the $\mathrm{p} 3$ domain (see Introduction and [5]). This domain corresponds to a NK6/Pax6positive domain from which originate $\mathrm{Hb}$ 9-positive cholinergic motor neurons and oligodendrocytes, the specification of both of these cells types being controled by olig2. This NK6/Pax6-positive domain also exists in Platynereis and, as in vertebrates, $\mathrm{Hb} 9$-positive cholinergic neurons emerge from this domain [35]. Pdu-Olig is, however, not expressed in this domain (it is expressed in the more medial NK6/NK2.2-positive domain) and is therefore much probably not involved in motor neurons specification. However its co-expression with $P d u$-ASH at some but not all stages of VNC formation, in the NKG/ NK2.2-positive domain, suggests it may contribute to the diversification of neural cell types from this domain. We suggest that different neural cell types may form in the prospective Platynereis VNC first from the $P d u-A S H / P d u$ Olig-positive cells and then from the Pdu-ASH-positive (Pdu-Olig-negative) cells. This suggestion is based on the fact that, in vertebrates, different combinations of neural bHLH have been shown to control the formation of different cell types from a single medio-lateral domain $[5,48]$.

Our data suggest that some neural bHLH genes are involved in neural subtype specification in Platynereis, like in vertebrates, and that this may therefore represent an ancestral feature of bilaterians. In insects, such as Drosophila, the specification functions of neural bHLH genes (and even some genes such as Olig) have been lost. This may be related to the fact that insects have evolved a divergent way to pattern their CNS: while in vertebrates and Platynereis the CNS is subdivided into large domains from which emerge specific neural cell types, insects have shifted to a mainly cell-to-cell-based process in which neuroblasts (even neighbouring ones) will express different combinations of developmental genes that control their identity.

\section{Conclusion}

We have identified, for the first time in a lophotrochozoan species, Platynereis dumerilii, orthologs of the most important neural bHLH genes known in vertebrates, including the Olig and NeuroD genes not found in Drosophila. We have performed a detailed analysis of the expression patterns of these Platynereis bHLH genes and we show that all these genes are expressed during neurogenesis. Our analysis suggests that the Platynereis bHLH genes have both proneural and neuronal specification functions, in a way more akin to the vertebrate situation than to that of Drosophila. Our data suggest that these functions are ancestral to bilaterians.

\section{Methods}

Breeding culture, embryo collection, whole mount in situ hybridization (WMISH), microscopy, and image processing Animals were obtained from a breeding culture established in Gif-sur-Yvette according to the protocol of Fisher and Dorresteijn [49]. Larvae collection and fixation, as well as WMISH, were done as previously described $[39,50]$. In the case of the double WMISH, one of the probes was revealed using tyramide signal amplification (fluorescent dye) and the other using the classical NBT/ BCIP reaction. The NBT/BCIP staining was visualized by reflection confocal laser scanning microscopy [39]. Labeled embryos picture Z-stacks were manually taken on a Leica bright-field microscope and Z-projection images were made using ImageJ $1.36 \mathrm{~b}$. Confocal pictures were taken on a Leica Sp2 confocal microscope and images were 3D reconstructed with Metamorph.

\section{Isolation of Platynereis bHLH genes and phylogenetic analyses}

Platynereis bHLH genes were identified by BLAST searches against an EST collection [34]. The coding sequences of the different genes were amplified using SMART ${ }^{\mathrm{TM}}$ RACE cDNA amplification procedures with gene-specific primers (whose sequences are available upon request). PCRproducts were TA cloned into the PCR2.1 vector (Invitrogen), sequenced on an $\mathrm{ABI}$ automated sequencer, and used as template to produce labeled antisense RNA probes for WMISHs. Accession numbers for the newly cloned genes are FM163169 to FM163172. 
Multiple sequence alignments were built using ClustalW [51] using a large set of bHLH domains derived from Simionato et al. [31] in addition to those encoded by the isolated Platynereis genes. Neighbour-joining (NJ) reconstructions were performed with the PAUP 4.0 program using the BioNJ algorithm and 10,000 bootstrap replicates [52,53]. Maximum likelihood (ML) analyses were performed with PHYML using the Jones, Taylor, Thornton (JTT) model of amino acid substitutions [54] and 150 bootstraps to assess the statistical reliability of the obtained internal branches. Bayesian inference was performed using the Markov chain Monte Carlo method as implemented in the MRBAYES (version 3) package [55]. We used the JTT substitution frequency matrix with among-sites rate variation modelled by means of a discrete $\gamma$ distribution with four equally probable categories. Two independent Markov chains were run, each containing 2,000,000 Monte Carlo steps. One out of every 250 trees was saved. The trees obtained in the two runs were meshed and the first $25 \%$ of the trees were discarded as 'burnin'. Marginal probabilities at each internal branch were taken as a measure of statistical support. All the alignments and the trees are available upon request.

\section{Authors' contributions}

ES participated in the cloning of the genes and carried out most in situ hybridizations; PK participated in the cloning of the genes and carried out some in situ hybridizations; ND carried out the double in situ hybridizations and confocal imaging; MLG participated in the cloning of the genes and in situ hybridizations; VL participated in BLAST searches and phylogenetic analyses; DA participated in the design of the study and provided essential sequence data and materials; MV conceived the study, participated in BLAST searches, performed the phylogenetic analyses, and drafted the manuscript. All authors have read and approved the final manuscript.

\section{Acknowledgements}

We thank Genoscope (Evry) for producing the Platynereis ESTs sequences. This work was supported by grants from the Agence National de la Recherche and the Ministère Français de la Recherche, through its $\mathrm{ACl}$ 'Jeunes chercheurs'(MV). ES and ND hold fellowships from the Ministère Français de la Recherche and PK a "Bourse pour Docteur-Ingénieur" from the CNRS. VL was financially supported by the Belgian Science Policy. We thank Guillaume Balavoine for support and the Imaging and Cell Biology facility of the IFR87 (FR-W225I) for expert support with confocal microscopy.

\section{References}

I. Vervoort M, Ledent V: The evolution of the neural basic HelixLoop-Helix proteins. ScientificWorldJournal 200I, I:396-426.

2. Bertrand N, Castro DS, Guillemot F: Proneural genes and the specification of neural cell types. Nat Rev Neurosci 2002, 3:517-530.

3. Gomez-Skarmeta JL, Campuzano S, Modolell J: Half a century of neural prepatterning: the story of a few bristles and many genes. Nat Rev Neurosci 2003, 4:587-598.
4. Quan $X$ J, Hassan B: From skin to nerve: flies, vertebrates and the first helix. Cell Mol Life Sci 2005, 62:2036-2049.

5. Guillemot F: Spatial and temporal specification of neural fates by transcription factor codes. Development 2007. I34:377I-3780.

6. Ledent $\mathrm{V}$, Paquet $\mathrm{O}$, Vervoort M: Phylogenetic analysis of the human basic helix-loop-helix proteins. Genome Biol 2002, 3:RESEARCH0030.

7. Hassan BA, Bellen HJ: Doing the MATH: is the mouse a good model for fly development? Genes Dev 2000, I 4: 1852-I865.

8. Fode C, Gradwohl G, Morin X, Dierich A, LeMeur M, Goridis C, Guillemot F: The bHLH protein NEUROGENIN 2 is a determination factor for epibranchial placode-derived sensory neurons. Neuron 1998, 20:483-494.

9. Ma Q, Chen Z, del Barco Barrantes I, de la Pompa JL, Anderson DJ: neurogenin $I$ is essential for the determination of neuronal precursors for proximal cranial sensory ganglia. Neuron 1998, 20:469-482.

10. Ma Q, Fode C, Guillemot F, Anderson DJ: Neurogenin I and neurogenin2 control two distinct waves of neurogenesis in developing dorsal root ganglia. Genes Dev 1999, 13:1717-1728.

II. Bush A, Hiromi Y, Cole M: Biparous: a novel bHLH gene expressed in neuronal and glial precursors in Drosophila. Dev Biol 1996, 180:759-772.

12. Gautier P, Ledent V, Massaer M, Dambly-Chaudiere C, Ghysen A: tap, a Drosophila bHLH gene expressed in chemosensory organs. Gene 1997, 191:15-21.

13. Ghysen A, Dambly-Chaudiere C: The specification of sensory neuron identity in Drosophila. Bioessays 1993, 15:293-298.

14. Skeath JB: At the nexus between pattern formation and celltype specification: the generation of individual neuroblast fates in the Drosophila embryonic central nervous system. Bioessays 1999, 21:922-931.

15. Bermingham NA, Hassan BA, Wang VY, Fernandez M, Banfi S, Bellen HJ, Fritzsch B, Zoghbi HY: Proprioceptor pathway development is dependent on Math I. Neuron 200I, 30:4II-422.

16. Gowan K, Helms AW, Hunsaker TL, Collisson T, Ebert PJ, Odom R, Johnson JE: Crossinhibitory activities of Ngn I and Math I allow specification of distinct dorsal interneurons. Neuron 200I, 31:219-232.

17. Helms AW, Johnson JE: Specification of dorsal spinal cord interneurons. Curr Opin Neurobiol 2003, 13:42-49.

18. Zhuang B, Sockanathan S: Dorsal-ventral patterning: a view from the top. Curr Opin Neurobiol 2006, 16:20-24.

19. Fode C, Ma Q, Casarosa S, Ang SL, Anderson DJ, Guillemot F: A role for neural determination genes in specifying the dorsoventral identity of telencephalic neurons. Genes Dev 2000, I 4:67-80.

20. Parras CM, Schuurmans C, Scardigli R, Kim J, Anderson DJ, Guillemot F: Divergent functions of the proneural genes Mashl and Ngn2 in the specification of neuronal subtype identity. Genes Dev 2002, 16:324-338.

21. Goridis C, Rohrer H: Specification of catecholaminergic and serotonergic neurons. Nat Rev Neurosci 2002, 3:53I-54I.

22. Pattyn A, Simplicio N, van Doorninck JH, Goridis C, Guillemot F, Brunet JF: Ascl IIMash I is required for the development of central serotonergic neurons. Nat Neurosci 2004, 7:589-595.

23. Mizuguchi R, Sugimori M, Takebayashi H, Kosako H, Nagao M, Yoshida S, Nabeshima Y, Shimamura K, Nakafuku M: Combinatorial roles of olig2 and neurogenin 2 in the coordinated induction of pan-neuronal and subtype-specific properties of motoneurons. Neuron 200I, 31:757-77I.

24. Novitch BG, Chen Al, Jessell TM: Coordinate regulation of motor neuron subtype identity and pan-neuronal properties by the bHLH repressor Olig2. Neuron 200I, 31:773-789.

25. Lee JE, Hollenberg SM, Snider L, Turner DL, Lipnick N, Weintraub H: Conversion of Xenopus ectoderm into neurons by NeuroD, a basic helix-loop-helix protein. Science 1995, 268:836-844.

26. Miyata T, Maeda T, Lee JE: NeuroD is required for differentiation of the granule cells in the cerebellum and hippocampus. Genes Dev 1999, I3:1647-1652.

27. Schwab MH, Bartholomae A, Heimrich B, Feldmeyer D, DruffelAugustin S, Goebbels S, Naya FJ, Zhao S, Frotscher M, Tsai MJ, Nave $\mathrm{K}$ : Neuronal basic helix-loop-helix proteins (NEX and BETA2/Neuro D) regulate terminal granule cell differentiation in the hippocampus. J Neurosci 2000, 20:3714-3724. 
28. Lu OR, Sun T, Zhu Z, Ma N, Garcia M, Stiles CD, Rowitch DH: Common developmental requirement for Olig function indicates a motor neuron/oligodendrocyte connection. Cell 2002, 109:75-86.

29. Zhou Q, Anderson DJ: The bHLH transcription factors OLIG2 and OLIGI couple neuronal and glial subtype specification. Cell 2002, 109:6I-73.

30. Muller T, Anlag K, Wildner H, Britsch S, Treier M, Birchmeier C: The bHLH factor Olig3 coordinates the specification of dorsal neurons in the spinal cord. Genes Dev 2005, 19:733-743.

31. Simionato E, Ledent V, Richards G, Thomas-Chollier M, Kerner P, Coornaert D, Degnan BM, Vervoort : Origin and diversification of the basic helix-loop-helix gene family in metazoans: insights from comparative genomics. BMC Evol Biol 2007, 7:33.

32. Philippe $H$, Telford $M$ J: Large-scale sequencing and the new animal phylogeny. Trends Ecol Evol 2006, 2 I:6 14-620.

33. Tessmar-Raible K, Arendt : Emerging systems: between vertebrates and arthropods, the Lophotrochozoa. Curr Opin Genet Dev 2003, 13:331-340.

34. Raible F, Tessmar-Raible K, Osoegawa K, Wincker P, Jubin C, Balavoine $G$, Ferrier $D$, Benes V, de Jong P, Weissenbach J, Bork P, Arend $D$ : Vertebrate-type intron-rich genes in the marine annelid Platynereis dumerilii. Science 2005, 3 10: | 325-1326.

35. Denes AS, Jekely G, Steinmetz PR, Raible F, Snyman H, Prud'homme $B$, Ferrier DE, Balavoine G, Arendt D: Molecular architecture of annelid nerve cord supports common origin of nervous system centralization in bilateria. Cell 2007, 129:277-288.

36. Fischer A, Dorresteijn A: The polychaete Platynereis dumerilii (Annelida): a laboratory animal with spiralian cleavage, lifelong segment proliferation and a mixed benthic/pelagic life cycle. Bioessays 2004, 26:3|4-325

37. Arendt D, Tessmar K, de Campos-Baptista MI, Dorresteijn A, Wittbrodt J: Development of pigment-cup eyes in the polychaete Platynereis dumerilii and evolutionary conservation of larva eyes in Bilateria. Development 2002, I29: I |43-I I54.

38. Graham $V$, Khudyakov J, Ellis P, Pevny: SOX2 functions to maintain neural progenitor identity. Neuron 2003, 39:749-765.

39. Jékely $G$, Arendt $D$ : Cellular resolution expression profiling using confocal detection of NBT/BCIP precipitate by reflection microscopy. Biotechniques 2007, 42:75I-755.

40. Kerner P, Zelada González F, Le Gouar M, Ledent V, Arendt D, Vervoort $M$ : The expression of a hunchback ortholog in the polychaete annelid Platynereis dumerilii suggests an ancestral role in mesoderm development and neurogenesis. Dev Genes Evol 2006, 216:821-828.

41. Rebscher N, Zelada-Gonzalez F, Banisch TU, Raible F, Arendt D: Vasa unveils a common origin of germ cells and of somatic stem cells from the posterior growth zone in the polychaete Platynereis dumerilii. Dev Biol 2007, 306:599-6II.

42. de Rosa R, Prud'homme B, Balavoine G: Caudal and even-skipped in the annelid Platynereis dumerilii and the ancestry of posterior growth. Evol Dev 2005, 7:574-587.

43. Ledent $V$, Vervoort M: The basic helix-loop-helix protein family: comparative genomics and phylogenetic analysis. Genome Res 200I, I I:754-770.

44. Hallam $S$, Singer $E$, Waring $D$, jin $Y$ : The C. elegans NeuroD homolog cnd-I functions in multiple aspects of motor neuron fate specification. Development 2000, 127:4239-4252.

45. Satou Y, Imai KS, Levine M, Kohara Y, Rokhsar D, Satoh N: A genomewide survey of developmentally relevant genes in Ciona intestinalis. I. Genes for bHLH transcription factors. Dev Genes Evol 2003, 21 3:213-22I.

46. Stollewerk A, Simpson P: Evolution of early development of the nervous system: a comparison between arthropods. Bioessays 2005, 27:874-883.

47. Skeath JB, Doe CQ: The achaete-scute complex proneural genes contribute to neural precursor specification in the Drosophila CNS. Curr Biol 1996, 6: I I 46- II 52.

48. Sugimori M, Nagao M, Bertrand N, Parras CM, Guillemot F, Nakafuku $M$ : Combinatorial actions of patterning and HLH transcription factors in the spatiotemporal control of neurogenesis and gliogenesis in the developing spinal cord. Development 2007, 134:1617-1629.

49. The Platynereis dumerilii Homepage [http://www.uni-gies sen.de/ gfl019/home/]
50. Tessmar-Raible K, Steinmetz PR, Snyman H, Hassel M, Arendt : Fluorescent two-color whole mount in situ hybridization in Platynereis dumerilii (Polychaeta, Annelida), an emerging marine molecular model for evolution and development. Biotechniques 2005, 39:460-464.

5I. Thompson JD, Higgins JD, Gibson TJ: CLUSTALW: improving the sensitivity of progressive multiple sequence alignment through sequence weighting, position-specific gap penalties and weight matrix choice. Nucleic Acids Res 1994, 22:4673-4680.

52. Gascuel O: BIONJ: an improved version of the NJ algorithm based on a simple model of sequence data. Mol Biol Evol I997, I 4:685-695.

53. Swofford DL: PAUP: Phylogenetic Analysis Using Parsimony (and Other Methods), Version 4. Sunderland, MA: Sinauer; 1998.

54. Guindon S, Gascuel O: A simple, fast, and accurate algorithm to estimate large phylogenies by maximum likelihood. Syst Biol 2003, 52:696-704.

55. Ronquist F, Huelsenbeck JP: MrBayes 3: Bayesian phylogenetic inference under mixed models. Bioinformatics 2003, 19:1572-1574.

Publish with Bio Med Central and every scientist can read your work free of charge

"BioMed Central will be the most significant development for disseminating the results of biomedical research in our lifetime. "

Sir Paul Nurse, Cancer Research UK

Your research papers will be:

- available free of charge to the entire biomedical community

- peer reviewed and published immediately upon acceptance

- cited in PubMed and archived on PubMed Central

- yours - you keep the copyright 